\title{
Experimental studies and correlation analysis of atmospheric factors affecting hexavalent chromium conglomerates with organic materials
}

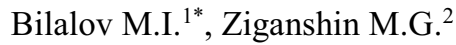 \\ ${ }^{1}$ LLC «Satellite of Safety», engineer, industrial engineering unit, Kazan, Russia \\ ${ }^{2}$ Kazan State Power Engineering University, Doctor of Technical Sciences, professor, Kazan, Russia
}

\begin{abstract}
Field testing of samples containing hexavalent chromium and organic compounds were carried out, which showed the possibility of intensifying the processes of their heat treatment using solar energy. The correlation analysis between various factors of atmospheric exposure and changes in the state of samples was performed. In the course of the study, an estimate was obtained for the wavelength ranges of the near-UV, visible and near-IR spectrum, within which photo-chemical reactions of hexavalent chromium reduction are possible. The temperature and hydrostatic pressure influence on the chemical reactions intensity in the sample was also assessed. Based on the data obtained, theoretical pre-conditions of energy efficiency of heat treatment process were formed, which should be verified by subsequent laboratory tests.
\end{abstract}

\section{Introduction}

Despite its high toxicity, hexavalent chromium is still widely used in many industries to protect products from corrosion and for decorative finishing. Chromium has become an important component of stainless steels, as well as a number of other alloys. In the aerospace and engineering industries are widely used products that have been galvanized with hexavalent chromium. But they will have a dark side. This is the toxicity of such production for both personnel and for residents living near the plant. Here the problem arises of choosing between the high toxicity of industrial waste and replacing of chrome plating with less toxic production, which leads to a decrease in product quality. The optimal solution is to develop new or/and improve existing methods for neutralizing waste containing hexavalent chromium.

In order to reveal the influence of atmospheric factors on conglomerates of hexavalent chromium with an organic film-forming substance (linseed oil oxol), field tests in Kazan and Naberezhnye Chelny, Republic of Tatarstan, Russia carried out [2]. The experiment involved 11 samples. Based on the data obtained during field tests, a correlation analysis was performed for the first sample. Based on the results of the study, theoretical suppositions are formulated that require further verification.

\section{Methods}

For the convenience and correctness of the analysis due to the large number of experimental data, 3 types of sample transformations were identified, which differed in the intensity of changes that occurred during the observation period. For Type 1, we have included such qualitative and/or semi-quantitative changes that are characterized by a change in the color and/or consistency of the sample during the time interval between two subsequent registrations, which averaged from 2 to 4 days. Type 1 was divided into two subtypes: 1.1 - subtype with "strong" and 1.2 - subtype with "weak" qualitative changes. At the same time, only such changes that were fixed as clearly objectively as the more intensive changes of sub-type 1.1, were classified to subtype 1.2. Type 2 included such semi-quantitative changes as increased color intensity and/or expansion of the area of change in the samples consistency, which could be noticed only after several registrations, and during the time interval between two consecutive registrations, no objectively changes could be detected.

In our work, type 3 included intervals of the state of samples which qualitative or semi-quantitative changes in its color and/or consistency could not be objectively detected in.

Figure 1 shows the character of the observed changes in the state of the samples during the whole period of the study, broken down into types of transformations.

\footnotetext{
*Corresponding author: marselbil@gmail.com
} 


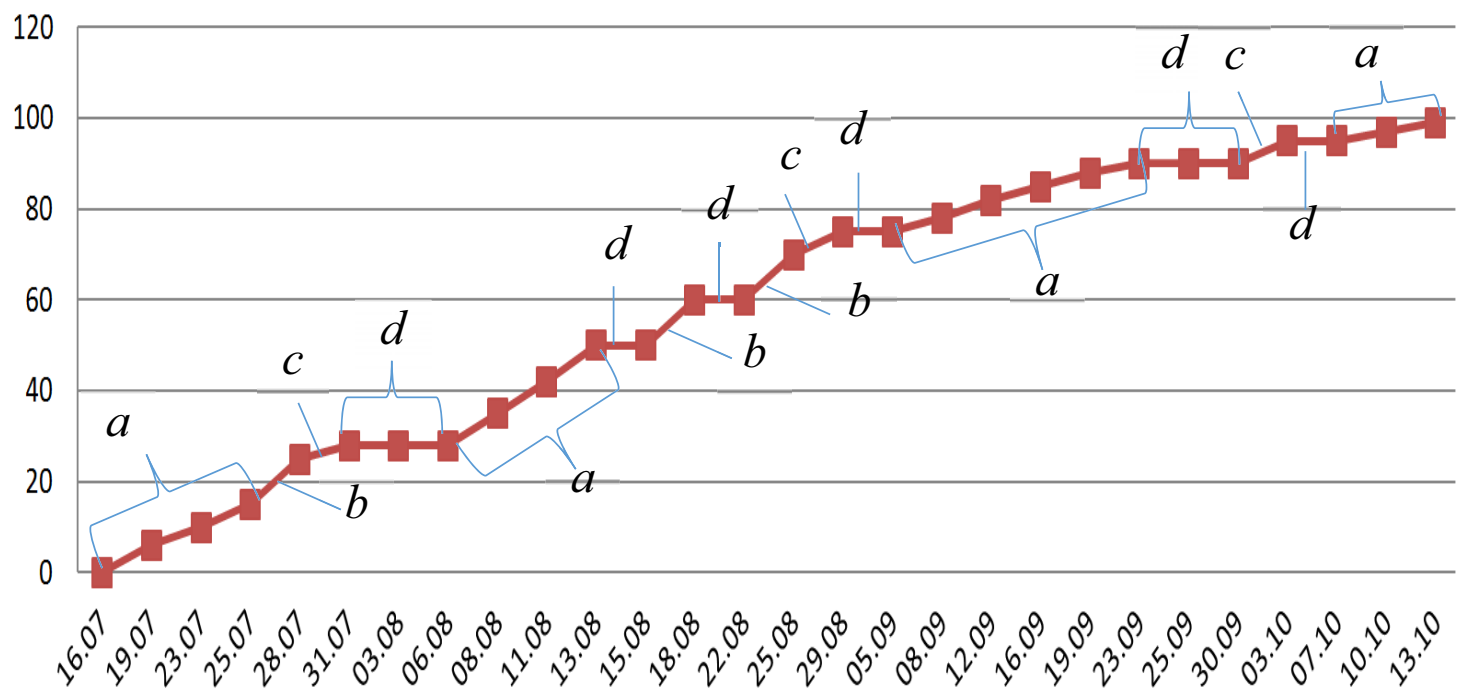

Fig. 1. Graph of observed changes in the state of the first sample and their types: $\mathrm{a}$ - type $2, \mathrm{~b}$ - subtype $1.1, \mathrm{c}$ - subtype $1.2, \mathrm{~d}$ - type 3 .

\section{Results and discussion}

Correlation analysis of graphs of changes in the state of samples was carried out on a number of parameters.

Figure 2 shows a correlation analysis of the graph of changes in the state of the first sample based on the daily outdoor temperature. Graphs of maximum and average daily temperatures can be used to indicate whether or not qualitative and semi-quantitative changes in sample correspond to changes in ambient temperature. The absence of correlation between them allows us to reliably state that fluctuations in the values of low thermal potential in the studied range of outdoor temperatures $9^{\circ} \mathrm{C} \div 32^{\circ} \mathrm{C}$ do not affect the transformation of samples.

The upper part of figure 2 shows graphs of changes in ambient temperature over the entire observed period. For example, the upper graph illustrates the dynamics of hourly temperature changes, and the lower graph shows the dew point temperature.

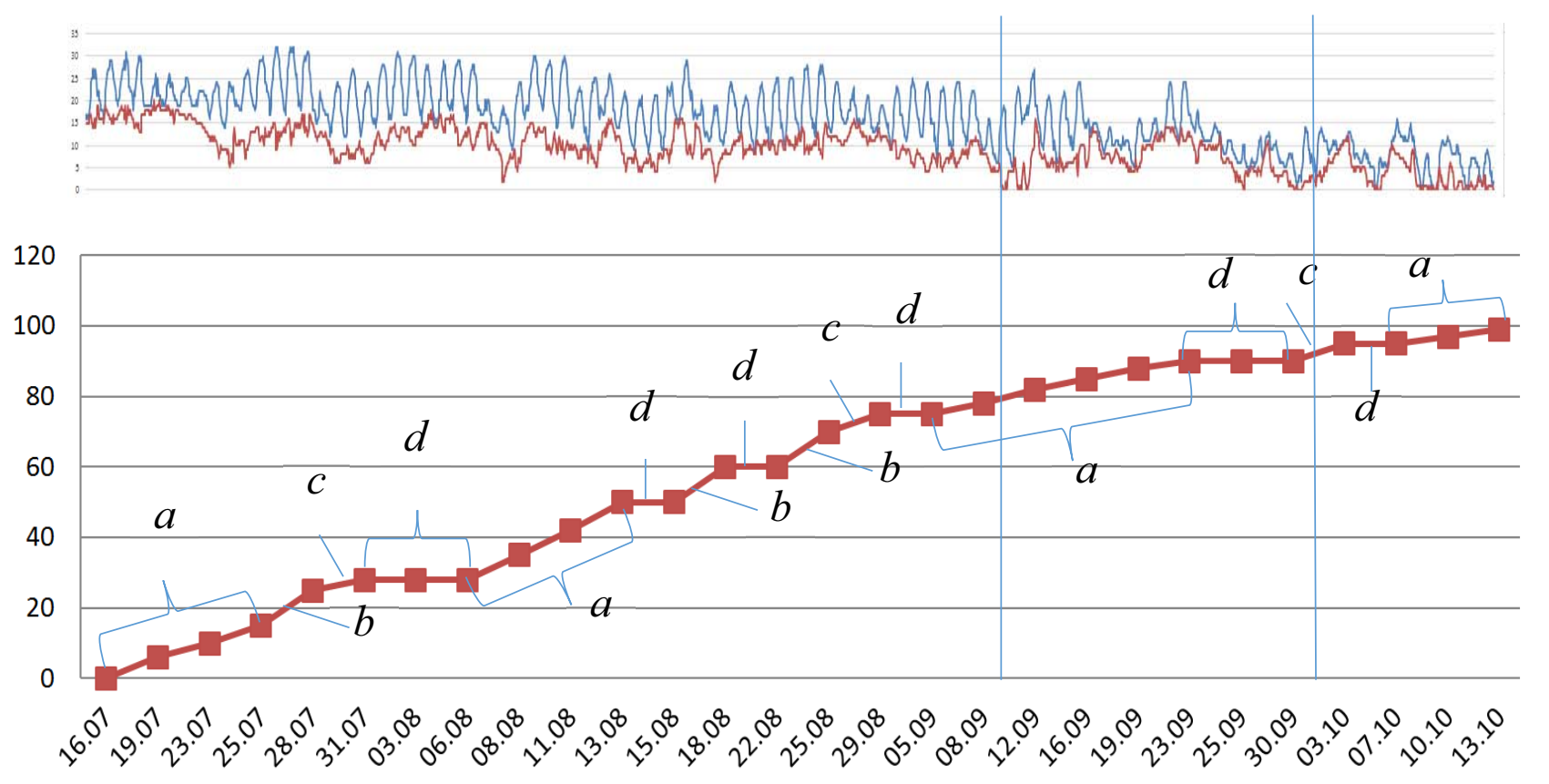

Fig. 2. Graphs of changes in ambient temperature and observed changes in the sample 1.

Changing the state of sample 1 at the beginning of the test corresponds to the interval of type 2 from 14.07 to 25.07 , during which the average of the maximum temperatures was $\sim 25^{\circ} \mathrm{C}$, and the maximum reached $31^{\circ} \mathrm{C}$. The observation interval from 25.07 to 26.07 of subtype 1.1 corresponds to the maximum outdoor 
temperature of $32^{\circ} \mathrm{C}$. Interval of subtype 1.2 from 28.07 to 31.07 corresponds to a decrease in the maximum air temperature from $31^{\circ} \mathrm{C}$ to $22^{\circ} \mathrm{C}$. Interval of subtype 3 from 31.07 to 06.08 occurred under conditions of transition from low temperatures to higher ones, to $28^{\circ} \mathrm{C}$ and higher. Further, the temperatures remain at the same level, and the changes enter type 2 from 06.08 to 13.08 . In this interval, the temperature drops sharply from $29^{\circ} \mathrm{C}$ to $19^{\circ} \mathrm{C}$, and then rises again to $30^{\circ} \mathrm{C}$ by 08.08 . However the sample changes are monotonous. Perhaps the response to these temperature surges was appears with delay: 13.08 to 15.08 by the nature of changes in the state of the sample type 3 and 15.08 to 18.08 by subtype 1.1 . During this period, the maximum temperature decreases from $30^{\circ} \mathrm{C}$ to $23^{\circ} \mathrm{C}$, rises to $26^{\circ} \mathrm{C}$ by 16.08 and again decreases to $20^{\circ} \mathrm{C}$ by 18.08 . The temperature rises to $29^{\circ} \mathrm{C}$ occurs on 21.08 , i.e. already after completion of changes in the sample of subtype 1.1 and their transition to type 3 from 18.08 to 22.08 . The reasons for the delay may consist in the imposition of other physical environmental factors on the sample, as well as the peculiarities of the chemistry of the sample transformation process. For example, in the presence of a chain reaction mechanism an increase or decrease in the break or branching ratios. For example, in the presence of a chain reaction mechanism, there may be an increase or decrease the chain branching or chain termination factor.

From 18.08 to 05.09 , the correlation is also objectively detected in the form of a trend of delaying changes in the quality of the first sample from changes in the maximum daily air temperature.

The interval from 05.09 to 23.09 is characterized by type 2 . In this interval, we objectively established the absence of any correlations.

It should also be noted that for the period from 14.07 to 29.08 , the first sample gained the main part (estimated $75 \%$ ) of objectively recorded changes. The rate of change over this period was $6.65 \times 10^{-2} \%$ per hour $(1.596 \%$ per day). In the subsequent period, the rate of change did not stop, but slowed down almost three times, to $2.31 \times 10^{-2} \%$ per hour $(0.56 \%$ per day). This confirms the influence of external temperature on the transformation of the first sample.

The next parameter was the sum of points of the daily UV indices. In figure 3, the upper part shows graphs of changes in the sum of points of the daily UV indices $\left(\mathrm{p}_{U V i}\right)$ and observed changes in the first sample. The upper graph illustrates the dynamics of changes in the sum of points of the daily UV indices $\left(\mathrm{p}_{U v i}\right)$. The lower graph shows the dynamics of changes in the maxima of the total daily UV indices.

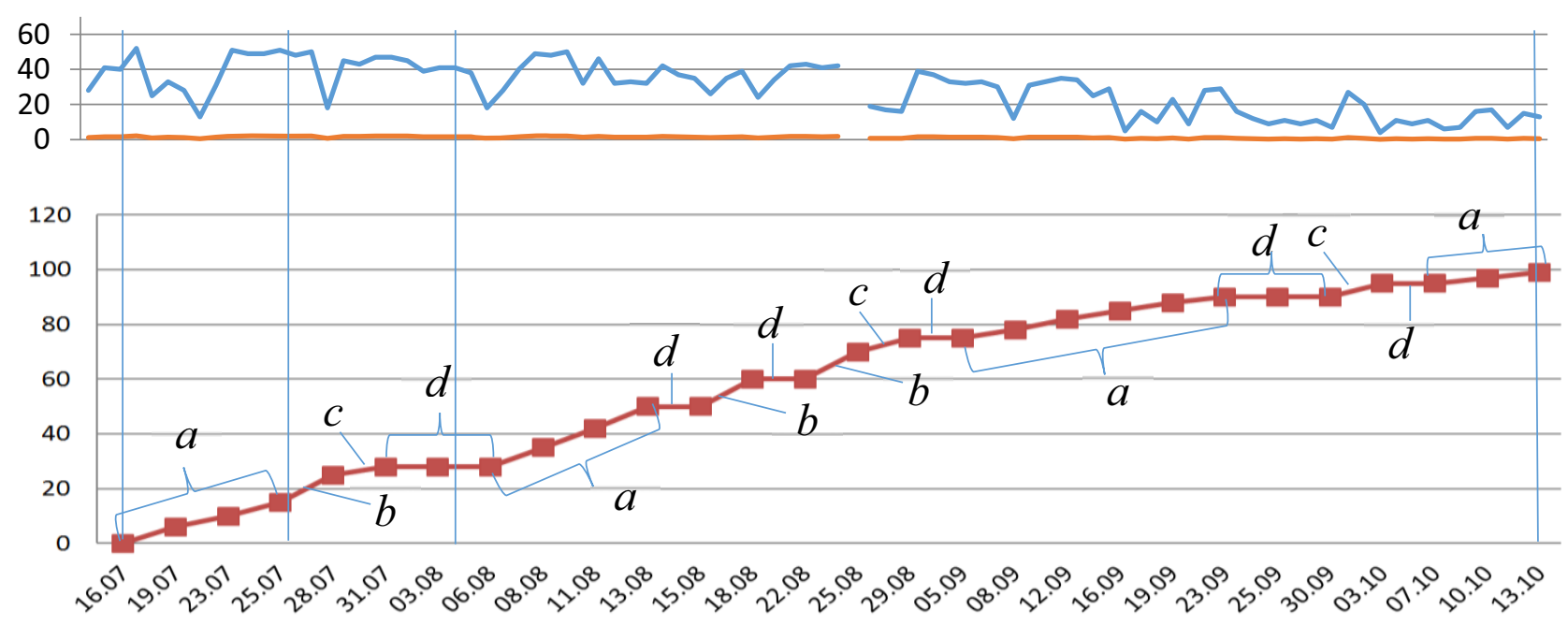

Fig. 3. Graphs of changes in the sum of points of the daily UV indices ( $\left.\mathrm{p}_{U V i}\right)$ and observed changes in the first sample.

In figure 3 , you can select 3 intervals with high, medium and low values of the maximum UV indices. In the first interval from 14.07 to 30.08 , the maximum indices are above $40 \mathrm{p}_{U V i}$, in the second interval from 01.09 to 14.09 - in the range from 30 to $40 \mathrm{p}_{U V i}$, and in the third one from 15.09 to 13.10 - below $30 \mathrm{p}_{U V i}$.

At the beginning of the experiment the change in the state of the first sample corresponds to the interval of type 2 from 14.07 to 25.07 , during which the maximum of the total daily UV indices increased from 28 to $52 \mathrm{p}_{U V i}$ by 17.07 , fell to 13 by 21.07 , and again increased to $51 \mathrm{p}_{U V i}$ by 23.07 . The observation interval from 25.07 to 26.07 of subtype 1.1 corresponds to the continuation of the reached maximum of the UV 49-51 indices $\mathrm{p}_{U V i}$. The period from 14.07 to 26.07 was the longest for the entire observation period for the effect of maximum $\mathrm{p}_{U V i}$ values. The maximum intensity of the first sample evolution from 25.07 to 28.07 correlates with both temperature and UV index changes.

From 28.07 to 31.07 , there is a sharp decline in the $\mathrm{UV}$ index from an almost maximum value of $51 \mathrm{p}_{U V i}$ 28.07 to $18 \mathrm{p}_{U V i} 29.07$, followed by an increase to 31.07 to $44 \mathrm{p}_{U V i}$. At the same time, the maximum air temperature decreased from $31^{\circ} \mathrm{C}$ to $22^{\circ} \mathrm{C}$. Both factors also correlate with a decrease in the intensity of changes in first sample to sub-type 1.2. 
Perhaps the previous sharp drop in UV indices was one of the reasons that from 31.07 to 06.08 there were no noticeable changes in the state of first sample for type 3, although the UV indices decreased gradually during this period (from 47 to $41 \mathrm{p}_{U V i}$ ) and, therefore, were quite high relative to the entire observation period. Taking into account the fact that the temperature also increased in this interval, we can talk about a trend of delay in the response of the first sample to a change (in this case, to a sharp drop) in the UV index.

It can be assumed that the action of sufficiently high UV indices during the previous 7 days caused the transition of changes in the state of the sample to type 2 from 06.08 to 13.08 . Changes in the state of the first sample in this interval are of the nature of a monotonous increase.

Perhaps the response of the first sample to this decrease in UV indices manifests in the form of a delayed transition of the nature of changes to type 3 from 13.08 to 15.08 , and the subsequent jump in UV indices - to subtype 1.1 from 15.08 to 18.08 . A similar trend in this observation interval was also detected earlier when analyzing the temperature graph. It can be assumed that the average level of UV from 13.08 to 18.08 supported by the rate of changes in the first sample related to process chemistry, for example, with the activation of the chain reaction mechanism that occurred due to the high UV levels in the previous interval from 11.08 to 13.08 . This could have caused changes from type 3 to subtype 1.1 from 15.08 to 18.08 .

The return of changes to type 3 from 18.08 to 22.08 could be associated with the imposition of a certain decrease in the UV indices from 19.08 to 22.08 of the impact of other physical environmental factors on the sample 1.

From 22.08 to 25.08 , the process of changing the state of sample 1 was carried out according to subtype 1.1 , which could be a delayed response to the peaks of UV indices over $40 \mathrm{p}_{U V i}$ from 15.08 and 19.08, since, despite the subsequent decrease, from 19.08 to 21.08 , the indices were at an average level of more than 30 $\mathrm{p}_{\text {UVi }}$. From 25.08 to 29.08 , the change in the state of sample 1 has moved to subtype 1.2. This slowdown in the process could be affected by the reduction of the indices 22.08 and 25.08 to 26 and $24 \mathrm{p}_{U V i}$.

It should be noted that in the interval from 22.08 to 29.08 , the correlation is objectively expressed as a steadily observed trend of delay in the nature of changes in the quality of sample 1 from changes in the UV indices. It should be noted also that by 29.08 , sample 1 had yield about $80 \%$ of the change in its state over the entire period.

From 29.08 to 05.09 , sample 1 was in the type 3 state. UV indices at the beginning of this interval sharply decreased from $42 \mathrm{p}_{U V i} 30.08$ to $19 \mathrm{p}_{U V i} 01.09$ and to $16 \mathrm{p}_{U V i} 03.09$, then also sharply increased to 39 $\mathrm{p}_{U V i}$ and $37 \mathrm{p}_{U V i} 05.09$, so the termination of the transformation of the state of sample 1 in this interval weakly correlates with the trend of delay in changing UV indices.

It is of particular interest that in this interval, as noted earlier, the absence of any correlations between the evolution of sample 1 and fluctuations in ambient temperature is objectively recorded. This allows us to give a preliminary assessment of the priorities of the action of environmental parameters on the development of processes in sample 1. It can be approximately concluded that at temperature maxima of less than $25^{\circ} \mathrm{C}$ and an average daily temperature below $20^{\circ} \mathrm{C}$, the main influence on the evolution of sample 1 is the effect of UV and possibly other factors, and at temperature maxima above $25^{\circ} \mathrm{C}$, fluctuations in the ambient temperature become a priority for the intensity of transformations in sample 1 .

The following steady state sample 1 by type 3 with at $03.10 \quad 07.10$ also correlates well with delayed exposure to extreme lowering of UV indices below 10 $\mathrm{p}_{U V i} 26.09-30.09$, and the ensuing change of state of sample 1-type 2 - also with delayed the impact of increased UV index up to $27 \mathrm{p}_{U V i} 01.10$. Here we note separately the steady state of sample 1-type 3 with at 03.10 07.10. In this interval, the absence of any correlations between the evolution of sample 1 and fluctuations in ambient air temperature is again objectively recorded, which again confirms the priority of the effect of UV index fluctuations on sample 1 at low temperatures, rather than temperature.

Thus, the graphs of changes in the state of sample 1 for most of the entire observation period correlate quite well with respect the trend of delay in the responses of their state to fluctuations in the total daily values of the UV indices.

Similarly, other parameters were analyzed for the first sample (relative humidity, wind speed, etc.). Thus, the correlation changes in the state of sample 1 with changes of the atmospheric pressure and wind speed was not objectively detected.

Analysis of the impact of environmental conditions on the evolution of sample 1, based on only one parameter, revealed some aspects of the process that are not uniquely identifiable. In some cases, an objective determination and rational explanation of the presence or absence of a trend in the evolution of sample 1 is possible if two or more factors simultaneously affect it. In this aspect, the expediency of using multiparametric indicators of the effect of the environment on the sample is considered. As part of the last stage, photos of the studied sample were analyzed for the presence of color and structural changes.

\section{Conclusion}

Based on the analysis of the research results, several preconditions for further research have been formed. Comparative analysis of photos of samples showed that the main confirmation of the passage of chemical reactions in the sample is a change in their color spectrum. For example, a sample that did not change its color during the experiment did not change also structurally. The results of the research also revealed the interrelation between hydrostatic pressure and qualitative transformation of samples. In the first few days conglomerate of dichromate and linseed oil oxol still retains fluidity. For better lighting at sun, the samples in the city of 
Kazan were installed at an angle. Figure 4 shows a diagram of the sample in a longitudinal section. As the diagram shows, the contents of the samples "slide" as a result of gravity, and a hydrostatic pressure gradient is formed in the longitudinal direction.

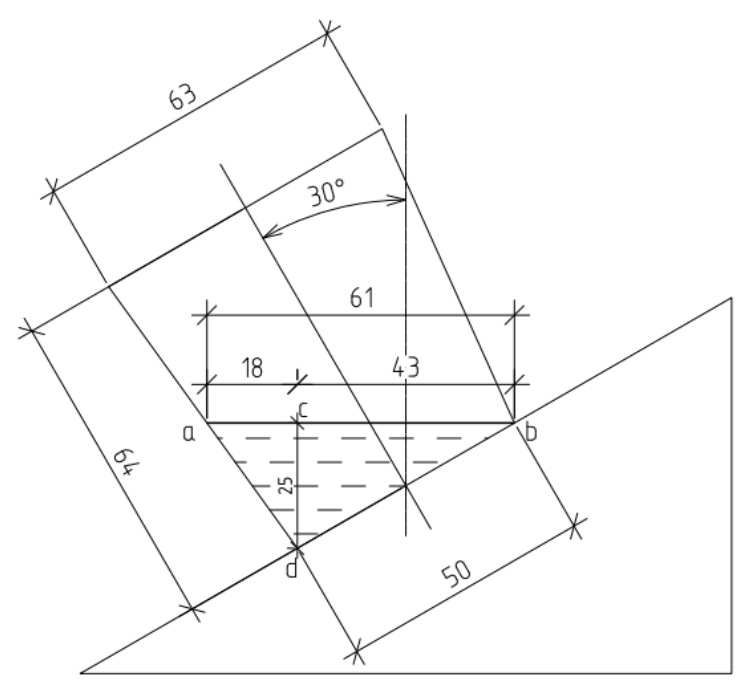

Fig. 4. Diagram of the sample in the longitudinal section.

The following is an example of estimating the pressure gradient of a sample whose height in the freshly prepared horizontal position " $\mathrm{h}$ " was about $10 \mathrm{~mm}$. Assuming the density of a sample of a mixture of dichromate and oil $\mathrm{p}=2500 \mathrm{~kg} / \mathrm{m}^{3}$, we can estimate the maximum hydrostatic pressure at $\mathrm{h} \sim 25 \mathrm{~mm} \mathrm{p}=613 \mathrm{~Pa}$. From here, the hydrostatic pressure gradient in the direction $\mathrm{cb}=43.3 \mathrm{~mm}$ will be $14.2 \mathrm{kPa} / \mathrm{m}$, and in the direction $\mathrm{ca}=18 \mathrm{~mm}$ will be $613 / 0,018=36.1 \mathrm{kPa} / \mathrm{m}$.

Thus, the estimates show that both the hydrostatic pressure itself and its gradient are significant variables. Thus, the hydrostatic pressure is almost twice as high as the pressure fluctuations $\Delta \mathrm{p}_{\mathrm{atm}}=(761-737)=24 \mathrm{~mm}$ of mercury, or $326 \mathrm{~Pa}$, for the entire observation period.

Another factor that can be used to detect the of chemical reactions happening is the change in sample density.

The results obtained made it possible to conceptualize the design of the pilot plant and the principles of further research on an energy-efficient method for heat treatment of waste containing hexavalent chromium, taking into account the specifics of the process identified during field tests of samples. The main factors affecting the reaction mechanism at the first stage are temperature and pressure in the sample, as well as UV radiation in the atmospheric range. For a detailed study of the effect of temperature on sample transformation, it is necessary to thermostat the volume in which the samples are placed.

For research the three-section boiler KChM-5 (iron boiler upgraded 5) on gas fuel was adopted as it meets the basic requirements for the design concept and industrial trial samples of heat treatment devices with utilization of used heat. Figure 5 shows the placement of experimental equipment.

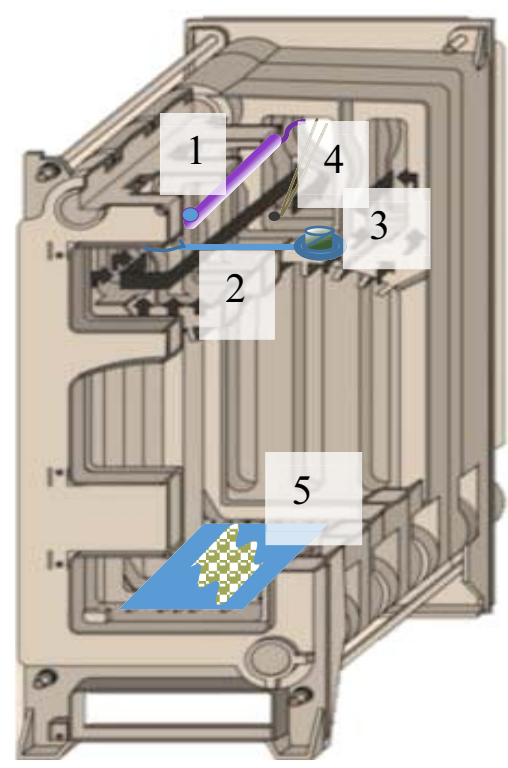

Fig. 5. Placement of experimental equipment in the furnace of a three-section KCHM - 5 boiler: 1 - UV lamp (OSRAM HNS S 11 W G23), 2 - metal stand, 3-bux with $\mathrm{K} 2 \mathrm{Cr} 2 \mathrm{O} 7$ breakdown on oil oxol, 4 - THA thermocouple, 5 - wood chips on a metal sheet.

In the boiler furnace, a UV lamp 1 (OSRAM HNS S 11 W G23) and a metal stand 2 for a heat-resistant graphite crucible with a sample 3 are attached to the sections. To ensure temperature control of the furnace, the boiler is filled with water to $85 \%(301)$. No gas was used in the trial. The burners were dismantled, and a metal sheet was placed on the floor, on which a small amount of wood chips was ignited 5. The flue gases were removed directly through the flue pipe. Also through it, a C-A thermocouple for temperature control was brought into the furnace. After adding fuel, when the temperature at the place where the thermocouple was installed reached $40^{\circ} \mathrm{C}$, a graphite crucible with a $\mathrm{K}_{2} \mathrm{Cr}_{2} \mathrm{O}_{7}$ sample on oil was installed on a metal stand fixed at the level of the thermo-couple on the boiler sections. Then rate of burning was increased to a temperature of $60^{\circ} \mathrm{C}$. This mode was maintained for 18 minutes, after which the fuel supply was stopped. After the temperature was lowered to $40^{\circ} \mathrm{C}$, the graphite crucible with the sample was removed from the furnace. In total, it was in the furnace for $\sim 45$ minutes. Its comparison with the control sample showed a significant difference, expressed in the intensification of the process and the appearance of local centers of darkening of the sample. In the field, this state of the sample was achieved in a few days. The conducted trial test has shown the validity of the proposed based field testing methods for intensifying the process of transformation of hexavalent chromium sample. However, it is obvious that this test does not allow us to quantify the contributions of temperature and UV radiation to the intensification of processes in the sample, which requires detailed studies, including the effect of the combined effect of temperature, UV radiation and pressure. 


\section{References}

1. GOST 12.1.007-76 «Occupational Safety Standards. Noxious substances. Classification and General Safety Requirements [electronic source] // [http://media.centrattek.ru/ckeditor_uploads/2015/0 6/29/vdnbro.pdf].

2. Bilalov M.I., Ziganshin M. G. (2019) Estimates of the influence of the solar irradiation spectrum energy on the intensity of heat treatment of solid waste with hexavalent chromium. IOP Conference Series: Materials Science and Engineering. Volume 481. 012044 - pp. 1-7. Doi:10.1088/1757899X/481/1/012044.

3. Garg K. K, Rawat P., Prasad B. (2015) Int J Water and Wastewater Treatment. - 1(1). - pp. 1-9.

4. Krynin A. G. and Khokhlov Yu. A. (2013) Optical characteristics of a thermally stabilized polyethylene terephthalate film used for functional glazing materials Aviation Materials and Technologies. - 4. - 31-4.
5. Mondal S. K. and Saha P. (2018) Chemical Engineering Research \& Design. - 132. - pp. 564583.

6. Owlad Mojdeh, Aroua Mohamed, Daud Wan, and Baroutian Saeid (2009) Water, Air \& Soil Pollution. - 200 (1-4). - pp. 59-77.

7. Serova V. N. (2010) Optical and other materials based on transparent polymers (Kazan: KSTU). - p. 540.

8. Speranskaya T. A. (1976) Optical properties of polymers (Leningrad: Chemistry) - p. 136.

9. Suleymanov M. Zh. (2006) Spectral characteristics of transparent coatings of solar collectors In the collection. of the XXI International Conference "Equations of state of matter" (Chernogolovka: IPCP RAS) 207-4.

10. Waste Electrical \& Electronic Equipment European Commission ('DG Environment'). Available at: http://ec.europa.eu/environment/ waste/weee/index_en.htm [Accessed 20 September 2020]. 